\title{
Software de Realidad Aumentada para la enseñanza- aprendizaje de la asignatura Informática en la Ingeniería Mecánica
}

\author{
Irisleydis Mayol Céspedes, José Antonio Leyva Regalón, José Antonio Leyva \\ Reyes \\ Departamento de Ciencias Básicas e Informática Aplicada, Universidad de Granma. \\ Bayamo, Cuba \\ \{imayolc, jaleyva, jleyvar\}@udg.co.cu
}

Resumen. En el presente trabajo se abordan los resultados de la aplicación de un software de Realidad Aumentada en la asignatura Informática de la carrera de Ingeniería Mecánica de la Universidad de Granma. El software contiene ejercicios relacionados con contenidos de la asignatura, con el principal propósito de estimular la motivación de los estudiantes. Se implementaron técnicas de interacción basadas en el uso de marcadores fiduciales y se diseñó una base de datos para el almacenamiento de las configuraciones de las escenas aumentadas. En un estudio realizado se planificaron y ejecutaron actividades prácticas y evaluativas para el trabajo individual y colectivo de los alumnos. Los resultados evidenciaron una incidencia positiva del software en el proceso de enseñanza-aprendizaje y un incremento de los conocimientos básicos para los temas restantes de la asignatura.

Palabras Clave: Realidad Aumentada, marcadores fiduciales, enseñanzaaprendizaje, software educativo.

\begin{abstract}
In the present work the results of the application of an Augmented Reality software in the Computer Science course of the Mechanical Engineering degree at the University of Granma, are given. The software contains exercises related to the contents of the subject matter, with the main purpose of stimulating students' motivation. Interaction techniques based on the use of fiducial markers were implemented and a database was designed to store the augmented scene configurations. In one study carried out, practical and evaluative activities were planned and executed for individual and group work of the students. The results evidenced a positive incidence of the software in the teaching-learning process and the increase in the basic knowledge of the students for the remaining themes of the subject matter.
\end{abstract}

Keywords: Augmented Reality, fiducial markers, teaching-learning, educational software. 


\section{Introducción}

El egresado de la carrera de Ingeniería Mecánica está preparado para diseñar, fabricar, operar y mantener máquinas, equipos, instalaciones, sistemas mecánicos y de transformación de la energía. Durante el período de formación de un ingeniero mecánico cubano, se contribuye al logro de un conocimiento profundo de las ciencias básicas y los fundamentos de la ingeniería, que le permitan un mayor campo de acción en la esfera social y científica.

Entre las disciplinas que conforman el Plan de Estudio de la carrera de Ingeniería Mecánica de las instituciones de la Educación Superior de Cuba, está la Informática, la cual proporciona al estudiante la capacidad de trabajar, comunicarse y aprender a través de las tecnologías de la información y las comunicaciones. Una de estas instituciones es la Universidad de Granma, en la cual se imparten contenidos relacionados con la ofimática, los sistemas de cómputos, la algoritmización y un lenguaje de programación de alto nivel en la asignatura Informática de la carrera de Ingeniería Mecánica.

El principal objetivo de la asignatura es garantizar los conocimientos y habilidades básicas del estudiantado para que logre un mayor desempeño en las restantes materias que conforman el Plan de Estudio. Los temas relacionados con la algoritmización y la programación desarrollan el pensamiento lógico del ingeniero y lo preparan para que sea capaz de resolver problemas de la profesión.

El estudio previo de conceptos y características de los sistemas operativos, hardware y redes de computadoras, así como la resolución de problemas a través del uso de diagramas de flujo, constituyen un vínculo importante para la comprensión del lenguaje de programación de la asignatura. En la enseñanza-aprendizaje de esos contenidos el colectivo de profesores de la asignatura emplea algunos medios didácticos como audiovisuales y multimedias de la plataforma virtual de la universidad.

La escasa disponibilidad de los medios didácticos impide el total cumplimiento de los objetivos de la asignatura, debido a que el estudiante no logra una amplia comprensión de los conceptos y la interrelación entre los componentes de hardware. Una práctica docente para minimizar el impacto negativo de dicha limitante es garantizar que dichos componentes resulten más tangibles para los estudiantes. Sin embargo, la insuficiencia física de ellos para llevar a cabo dicha actividad impide el logro de una mayor calidad en la enseñanza-aprendizaje de este tema.

La resolución de problemas con los diagramas de flujos se ve afectada, en ocasiones, por la desmotivación del estudiante frente a la novedad y la complejidad del contenido, y el tiempo necesario que le toma familiarizarse con alguna herramienta para la diagramación. Estas limitantes precisan de medios que permitan mejorar la enseñanza-aprendizaje de la asignatura Informática de la Ingeniería Mecánica de la Universidad de Granma, para consecuentemente, incrementar las competencias informáticas del estudiante para un mejor desempeño en el resto de las materias de la carrera.

Los diversos procesos de enseñanza-aprendizaje en la Educación Superior han sido apoyados por el uso de programas informáticos basados en la Realidad Aumentada (RA), con el fin de incrementar la motivación de los estudiantes mediante una perspectiva diferente de representar contenidos, fenómenos y situaciones simples o 
complejas de abordar en un escenario docente [1-4]. En la RA un entorno físico es enriquecido por información virtual en tiempo real y se facilitan múltiples vías de interacción que, por lo general, involucran métodos naturales [5-7].

En este trabajo se exponen los resultados de la aplicación de un software de Realidad Aumentada, desarrollado por el colectivo de profesores de la asignatura Informática de la carrera de Ingeniería Mecánica de la Universidad de Granma, con el propósito de mejorar el proceso de enseñanza-aprendizaje. Las actividades del software están enfocadas a minimizar los impactos de las limitantes abordadas mediante escenas aumentadas que motiven al estudiante a la comprensión y la práctica de los contenidos recreados en el programa.

\section{Interacción en las escenas aumentadas}

La interacción en la RA constituye una de sus principales potencialidades para la manipulación de objetos 3D. Dado que el usuario es parte de la escena aumentada, la resultante de enriquecer con información virtual un entorno real, los métodos de interacción abarcan no solo el uso de periféricos, sino herramientas no electrónicas y de fácil confección.

Una de estas herramientas es el marcador fiducial de la librería ARToolKit, el cual constituye una tarjeta de papel con una imagen específica para ser reconocida por algoritmos visión por computador [8]. Los marcadores fiduciales son reconocidos mediante el video proporcionado por una cámara física para establecer un sistema de referencia para la información virtual de la escena aumentada.

Para la interacción en las escenas aumentada del software desarrollado, se implementaron tres técnicas basadas en el uso de los marcadores fiduciales. La primera es la oclusión de marcadores [9], con la cual se simuló un panel de tres botones para seleccionar objetos 3D y aplicar acciones específicas. La segunda consiste en un cubo con marcadores [10,11], empleado como herramienta para trasladar, rotar y escalar un elemento virtual seleccionado. Por último, se utilizaron marcadores independientes para generar eventos cuando ocurre una proximidad entre ellos [12].

La aplicación de RA fue realizada con la librería osgART [13], la cual integra ARToolKit con el motor gráfico OpenSceneGraph. Todas las escenas están agrupadas en una estructura de datos de árbol y la organización correspondiente de cada objeto virtual en la escena de RA, se encuentra en una base de datos relacional de SQLite [14].

\section{Descripción del software}

El programa constituye una aplicación de escritorio que requiere de una cámara para la captura del video en vivo y los marcadores fiduciales para la manipulación de la información virtual. El software contiene una serie de funcionalidades desarrolladas con el framework Qt [15], para que un profesor gestione las distintas actividades que se exponen en la Tabla 1. 
Tabla 1. Actividades del software de Realidad Aumentada

\begin{tabular}{lll}
\hline Tipo de Actividad & Herramienta de Interacción & Usuario \\
\hline Presentación de componentes & Cubo, panel de botones & Un profesor \\
Evaluación de componentes & Cubo, panel de botones & Un estudiante \\
Práctica de Diagrama de Flujo & $\begin{array}{l}\text { Marcadores independientes, panel } \\
\text { de botones }\end{array}$ & Un profesor \\
Evaluación de Diagrama de Flujo & $\begin{array}{l}\text { Marcadores independientes, panel } \\
\text { de botones }\end{array}$ & Estudiantes
\end{tabular}

En la presentación de componentes el profesor puede mostrar un conjunto de elementos virtuales relacionados con las partes de un hardware específico (Figura 1). La presentación está compuesta por uno o varios conjuntos de objetos $3 \mathrm{D}$ que son configurados previamente por el docente y asociados a un marcador de referencia que contiene el sistema global de coordenadas.

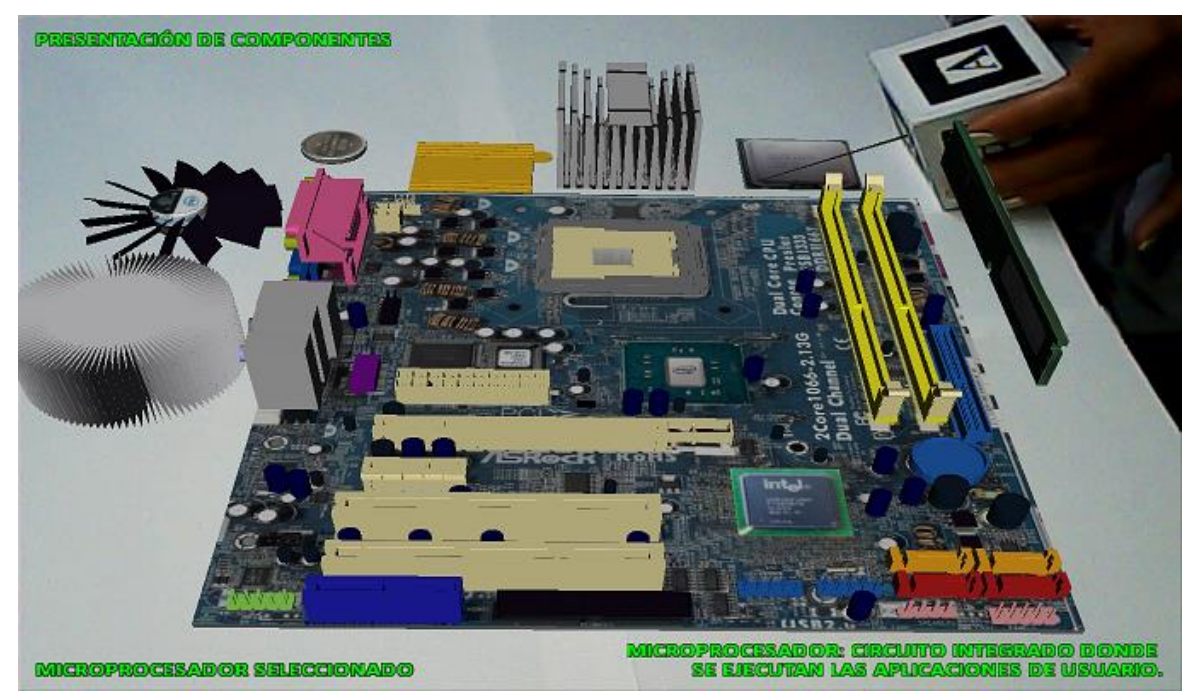

Figura 1. Interacción en la presentación de componentes.

Los marcadores fiduciales independientes entre sí en la práctica de diagrama de flujo, contienen figuras 3D relacionadas con los símbolos de representación de condicional, entrada y salida de datos, proceso, inicio y fin (Figura 2). En la construcción de un diagrama el docente selecciona y coloca un marcador con un símbolo determinado próximo a otro para establecer una relación. Esta proximidad genera una línea de flujo que conecta a los símbolos dando respuesta secuencialmente a la problemática planteada. 


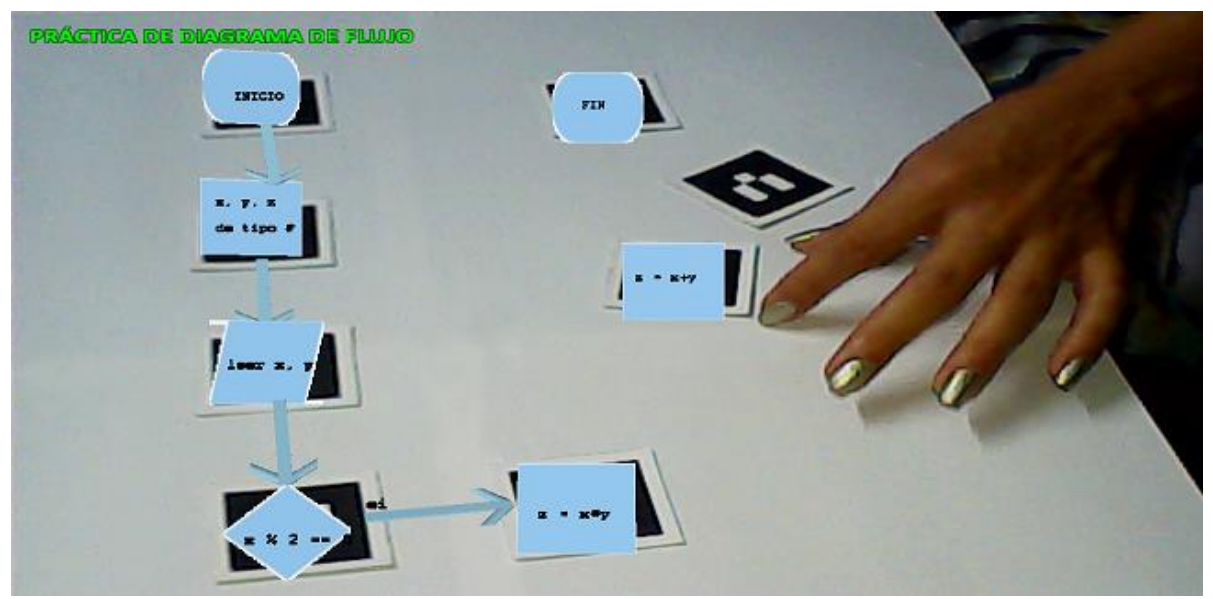

Figura 2. Interacción en la práctica de diagrama de flujo.

La actividad de evaluación de componentes tiene como objetivo que un estudiante responda preguntas relacionadas con las características de los elementos que se muestran. Algunas de las preguntas son respondidas a través del panel de botones y otras con el cubo cuando se requiera seleccionar un elemento acorde a las características de una pregunta para ser situado en un área específica de la escena aumentada. Esta última propiedad facilita al docente planificar situaciones como el ensamble de piezas virtuales, donde el estudiante hace coincidir la organización de los objetos similar al hardware real.

La evaluación de un diagrama está en dependencia de una problemática definida donde pueden participar uno o varios estudiantes en la resolución. Para configurar cualquiera de las actividades de la Tabla 1, el profesor dispone de formularios con las opciones necesarias para seleccionar elementos virtuales disponibles en el directorio del programa o cargar nuevos desde una fuente externa. Al mismo tiempo, puede establecer los conjuntos de componentes y el orden en que serán presentados, o introducir las preguntas y las correspondientes respuestas para la modalidad de evaluación.

La planificación de una evaluación de diagrama de flujo requiere que el profesor proponga un diagrama de solución a un problema a resolver. Luego, el programa genera en los marcadores todos los símbolos de la propuesta para que el estudiante construya posteriormente la solución.

\section{Resultados y discusión}

En la interacción con el software participaron 27 estudiantes de la carrera de Ingeniería Mecánica durante tres encuentros del curso académico 2018-2019. Para el caso de estudio, el colectivo de la asignatura diseñó una presentación de componentes de hardware de computadora y tres prácticas de diagrama de flujo de estructuras secuencial, alternativa y repetitiva. El diseño tuvo como finalidad familiarizar a los estudiantes con la RA y las herramientas de interacción. 
En la primera mitad del primer encuentro, los profesores realizaron la presentación y la simulación del ensamble de los componentes fundamentales de una placa madre con el uso del cubo. En la segunda mitad, los estudiantes interactuaron con los elementos virtuales en la escena aumentada realizando la selección y colocación de las piezas similar a su ubicación en una placa real.

De igual forma, en el segundo encuentro se realizaron las prácticas de diagrama de flujo con énfasis en la resolución de los problemas mediante el trabajo en equipos. El intercambio de ideas y el uso de los marcadores para resolver los ejercicios de forma interactiva, condicionaron un espacio educativo de entretenimiento que favoreció la motivación de los estudiantes.

Previo a los tres encuentros, se realizó una evaluación escrita para establecer un punto de partida del nivel de conocimiento de los estudiantes en los contenidos que se abordaron en el estudio. Para el tercer encuentro, se desarrollaron evaluaciones de forma individual empleando las técnicas de interacción de RA implementadas.

Una vez concluidos los ejercicios prácticos y evaluativos en el software se efectuó una segunda evaluación escrita para determinar la incidencia del contenido tratado con la RA. En la Figura 3 se muestran los respectivos promedios de las tres evaluaciones realizadas en el estudio en base a 5 puntos.

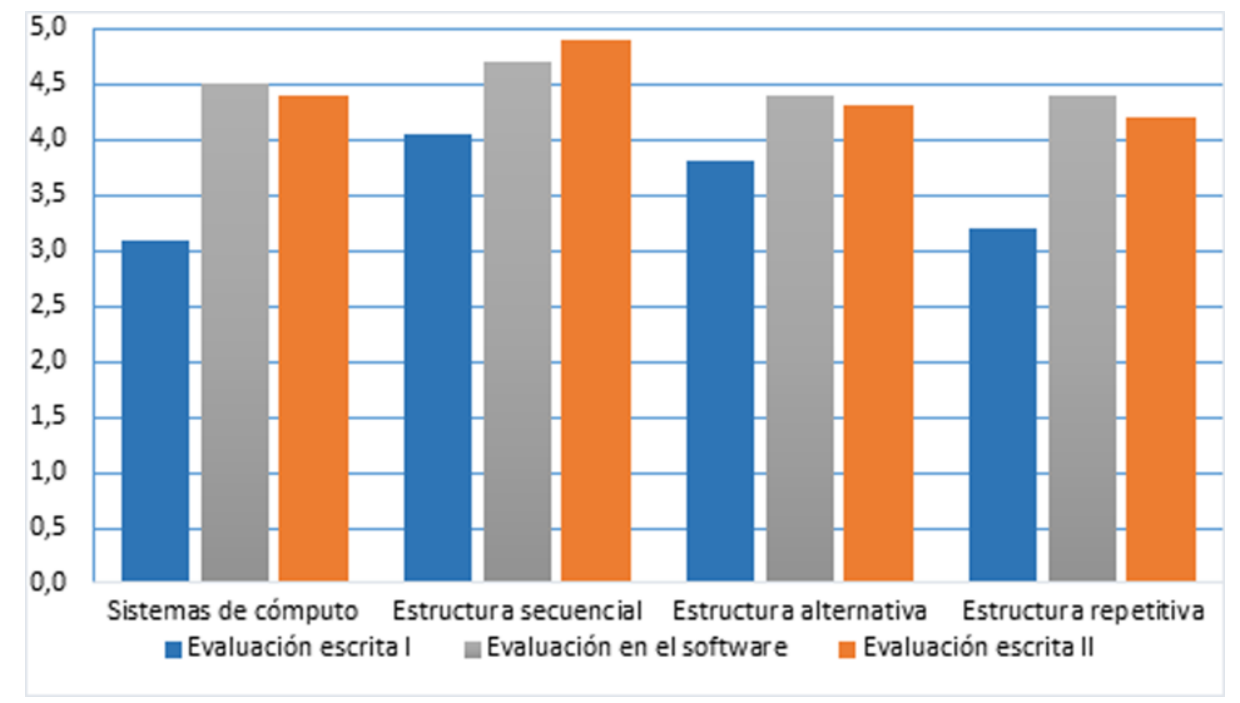

Figura 3. Promedio de evaluaciones de las actividades en el caso de estudio.

En la evaluación con el programa de RA se emplean diversos elementos de reconocimiento para la elección de la respuesta. Esto le proporciona al estudiante resultados superiores en la solución de los ejercicios, con mayor motivación y concentración en las actividades de aprendizaje.

La diferencia entre las evaluaciones escritas evidencia que la propuesta de RA favorece en la mayoría de los estudiantes la asimilación del contenido abordado. Consecuentemente, se fortalecen los conocimientos básicos para los temas restantes de la asignatura. 
El sistema de puntuación tiene en cuenta los errores que comete cada alumno y el tiempo en responder las preguntas o ejecutar las tareas específicas de interacción. El seguimiento de los errores permitió al programa mantener una adecuada retroalimentación con el estudiante sobre su desempeño en los ejercicios. De esta forma, el software ayudó a los alumnos que presentaban dificultades al resolver las actividades propuestas.

La rapidez con la que un estudiante completa cada actividad fue contabilizada en una variable de tiempo invisible en cualquiera de las escenas aumentadas. Esta característica permitió al sistema disponer de una bonificación individual para facilitar pistas al alumno en la resolución un ejercicio.

En el estudio, la bonificación constituyó un elemento significativo que influyó sobre la motivación del estudiante. El almacenamiento de todos estos paramentos fue propicio en el posterior análisis del progreso de cada alumno. Ello facilitó al docente dónde y cómo trabajar en el incremento de los resultados individuales del estudiantado.

\section{Conclusiones}

A través de la aplicación del software desarrollado, se logró una influencia sobre la estimulación de los estudiantes hacia el estudio de la asignatura. Lo anterior se evidenció en los resultados obtenidos de un caso de estudio en un escenario docente real, con estudiantes de la carrera de Ingeniería Mecánica de la Universidad de Granma.

El estudio permitió constatar las múltiples ventajas que permite la Realidad Aumentada para recrear ambientes educativos que favorezcan el proceso de enseñanza-aprendizaje. Las técnicas de interacción implementadas posibilitaron el trabajo individual y colectivo de los estudiantes, creándose un espacio de retroalimentación entre ellos y el sistema.

El almacenamiento en una base de datos de la evaluación y otros parámetros de los ejercicios computarizados fueron determinantes en la toma de decisiones, para el diseño de nuevas estrategias en el proceso docente. Cabe destacar, que el software presentado constituye el inicio de un proyecto más ambicioso para incrementar la calidad de la enseñanza-aprendizaje de la asignatura. Por tanto, se continuará su desarrollo para la planificación y evaluación automática de nuevas actividades que potencien la motivación del estudiantado.

\section{Referencias}

1. Dodge, J.C.M., De León, I.S.: Uso de realidad aumentada para enseñanza de conceptos básicos de física mecánica. Ingeniare. (12), 11-26 (2012). doi: https://doi.org/10.18041/1909-2458/ingeniare.12.631.

2. Ierache, J.S., et al.: Herramienta de Realidad Aumentada para facilitar la enseñanza en contextos educativos mediante el uso de las TICs. Revista Latinoamericana de Ingeniería de Software. 2(6), 365-368 (2014). doi: https://doi.org/10.18294/relais.2014.365-368. 
3. Alvarez-Marin, A., Castillo-Vergara, M., Pizarro-Guerrero, J., Espinoza-Vera, E.: Realidad aumentada como apoyo a la formación de ingenieros industriales. Formación Universitaria. 10(2), 31-42 (2017). doi: http://dx.doi.org/10.4067/S0718-50062017000200005

4. Ruiz, H.A.C., Jiménez, F.Y.M., Barón, M.J.S.J.E.y.c.: Realidad aumentada (RA): aplicaciones y desafíos para su uso en el aula de clase. Revista Educación y ciudad. (35), 137-148 (2018). doi: https://doi.org/10.36737/01230425.v0.n35.2018.1969.

5. Barrow, J., Forker, C., Sands, A., O'Hare, D., Hurst, W.: Augmented reality for enhancing life science education. In: Hurst, W., Bruni, V. (eds) VISUAL 2009: The Fourth International Conference on Applications and Systems of Visual Paradigms. Rome, Italy (2019).

6. Cabero-Almenara, J., Barroso-Osuna, J.: The educational possibilities of Augmented Reality. Journal of New Approaches in Educational Research. 5(1), 44-50 (2016). doi: https://dx.doi.org/10.7821/naer.2016.1.140.

7. Nizam, S.S.M., et al.: A review of multimodal interaction technique in augmented reality environment. International Journal on Advanced Science Engineering Information Technology. 8(4-2), 1460-1469 (2018). doi: http://dx.doi.org/10.18517/ijaseit.8.4-2.6824.

8. Rabbi, I., Ullah, S., Javed, M., Zen, K.: Analysing the attributes of fiducial markers for robust tracking in augmented reality applications. International Journal of Computational Vision Robotics. 7(1-2), 68-82 (2017). doi: https://doi.org/10.1504/IJCVR.2017.081238.

9. Garrido-Jurado, S., Muñoz-Salinas, R., Madrid-Cuevas, F.J., Marín-Jiménez, M.J.: Automatic generation and detection of highly reliable fiducial markers under occlusion. $\begin{array}{lllll}\text { Pattern Recognition. } & 47(6), & 2280-2292 & \text { (2014). doi: }\end{array}$ https://doi.org/10.1016/j.patcog.2014.01.005.

10. Zhou, Z., Cheok, A.D., Tedjokusumo, J., Omer, G.S.: wIzQubesTM-a novel tangible interface for interactive storytelling in mixed reality. The International Journal of Virtual Reality. 7(4), 9-15 (2008).

11. Rabbi, I., Ullah, S.: 3D Model Visualization and Interaction Using a Cubic Fiducial Marker. In: De Paolis, L.T., Mongelli, A. (eds) Augmented and Virtual Reality. AVR 2014. Lecture Notes in Computer Science. vol. 8853, pp. 381-393 Cham: Springer (2014). doi: https://doi.org/10.1007/978-3-319-13969-2_28.

12. Billinghurst, M., Grasset, R., Seichter, H.: Tangible interfaces for ambient augmented reality applications, in Human-Centric Interfaces for Ambient Intelligence. Aghajan, H., López-Cózar Delgado, R., Augusto, J.C., (eds), Elsevier. 281-302 (2010)

13. Looser, J., Grasset, R., Seichter, H., Billinghurst, M.: OSGART-A Pragmatic Approach to MR. In: 5th IEEE and ACM International Symposium on Mixed and Augmented Reality (ISMAR 2006): Industrial Workshop. Santa Barbara, CA, USA (2006).

14. Owens, M., Allen, G.: The Definitive Guide to SQLite. Apress (2010)

15. Rischpater, R.: Application development with qt creator. Packt Publishing Birmingham (2013) 